\title{
Dissonância cognitiva e consumo sustentável: uma revisão sistemática da literatura
}

Adilson Mueller

Mestrando no Programa de Pós-Graduação em Administração da Escola de Negócios da Pontifícia Universidade Católica do Rio Grande do Sul (PUCRS). Especialista em Gestão e Inteligência Competitiva pela PUCRS. Graduado em Filosofia e em Administração de Empresas pela PUCRS, Rio Grande do Sul, Brasil adilsonmueller@yahoo.com.br

Cláudio Damacena

Professor do Programa de Pós-Graduação em Administração da PUCRS

Dr. em Administração pela Universidade de Córdoba (Espanha)

Bacharel em Economia, Rio Grande do Sul, Brasil

damacena.claudio@gmail.com

Felipe Vidor Vaz

Graduando em Administração de Empresas pela Escola de Negócios da Pontifícia Universidade Católica do Rio Grande do Sul, Rio Grande do Sul, Brasil

vaz.felipe@gmail.com

Editor Científico: José Edson Lara

Organização Comitê Científico

Double Blind Review pelo SEER/OJS

Recebido em 22.09.2017

Aprovado em 20.12.2017 


\section{Resumo}

O estudo da teoria da dissonância cognitiva relacionada ao consumo sustentável é relativamente escasso. Nesse sentido, este artigo busca identificar e descrever as diferentes formas utilizadas pelas pessoas para lidar com a inconsistência entre atitudes e comportamentos relacionadas ao consumo sustentável. Para atender ao objetivo da pesquisa, utilizou-se o método da revisão sistemática da literatura. Os resultados evidenciam que as pessoas tendem a lidar com a tensão gerada pela dissonância cognitiva em relação ao consumo sustentável por meio do comprometimento, influência social, feedback, informações sobre produtos sustentáveis, negação e supressão de culpa, compensação, justificação, normas morais, benefícios percebidos versus custos/barreiras e semelhança comportamental. Esses achados aprofundam o entendimento de que substituir, esquecer ou adquirir novas cognições resolvem a dissonância e especificam detalhadamente um conjunto de justificativas presentes no comportamento do consumidor ao lidar com o fenômeno.

Palavras-chave: Dissonância cognitiva; Consumo sustentável; Comportamento do consumidor.

\section{Cognitive dissonance and sustainable consumption: a systematic review of the literature}

\section{Abstract}

The study of the theory of cognitive dissonance related to sustainable and relatively scarce consumption. In this sense, this article seeks to identify and describe how different ways by people to deal with an inconsistency between attitudes and behaviors related to sustainable consumption. To meet the research goal, use the systematic literature review method. The results show that as people tend to deal with the tension generated by cognitive dissonance in relation to sustainable consumption through commitment, social influence, feedback, information on sustainable products, negation and suppression of guilt, compensation, justification, moral norms, benefits perceived versus cost / barriers and behavioral mitigation. These findings deepen the understanding that replacing, forgetting or acquiring new cognitions solves a dissonance and specifies in detail a set of justifications present without consumer behavior in dealing with the phenomenon.

Key-words: Cognitive dissonance; Sustainable consumption; Consumer behavior. 


\section{Disonancia cognitiva y el consumo sostenibles: una revisión sistemática de la literatura}

\section{Resumen}

El estudio de la teoría de la disonancia cognitiva relacionada con el consumo sostenible y relativamente escaso. En este sentido, este artículo busca identificar y describir como diferentes formas por personas para lidiar con una inconsistencia entre actitudes y comportamientos relacionados con el consumo sostenible. Para atender al objetivo de la investigación, se utiliza el método de la revisión sistemática de la literatura. Los resultados evidencian que como las personas tienden a lidiar con la tensión generada por la disonancia cognitiva en relación al consumo sostenible a través del compromiso, la influencia social, la retroalimentación, la información sobre productos sostenibles, la negación y la supresión de la culpa, la compensación, la justificación, las normas morales, los beneficios percibidos frente a costos / barreras y mitigación conductual. Estos hallazgos profundizan el entendimiento de que sustituir, olvidar o adquirir nuevas cogniciones resuelven una disonancia y especifican detalladamente un conjunto de justificaciones presentes sin comportamiento del consumidor al tratar con el fenómeno.

\section{Introdução}

Existe uma tendência natural dos indivíduos de se comportarem de maneira semelhante com o que pensam (Thogersen, 2004), ou seja, procura-se atingir um estado harmonioso, consistente consigo ou nas interações com outros (Rodrigues, 1969). Diversas teorias explicam essa tendência de comportamento social e diferenciam-se na maneira como definem o termo consistência. Durante o auge das teorias da consistência cognitiva, uma teoria trouxe um elemento novo, diferente das demais teorias: a ideia de dissonância e consonância entre cognições. Com quase 60 anos de existência, a importante contribuição para essa tendência de comportamento (Teoria da Dissonância Cognitiva, segundo Festinger, 1957) resistiu a muitas experimentações, críticas e proposições e continua a oferecer uma explicação para o comportamento humano e integrar muitos outros paradigmas e teorias, como "Free choice", "Induced compliance", Effort justification", "Selfconsistency", "Self-affirmation", "New look" e "Action-Base Model Of Dissonance" (Harmon-Jones \& Harmon-Jones, 2007).

É sabido que a maioria das pessoas demonstra ter atitudes pró-ambientais, mas o comportamento, entretanto, não é coerente com essas atitudes (Verplanken \& 
Roy, 2016). Quando as pessoas são confrontadas com essa incoerência, ocorrem os conflitos cognitivos que podem ser resolvidos, portanto, com justificativas, alteração de atitudes, comportamentos ou ambos (Harmon-Jones, 2012).

Este artigo traz a análise de estudos que tratam da dissonância no consumo sustentável e descreve as principais variáveis que explicam como as pessoas lidam com esse fenômeno. São elas: comprometimento (Beretti, Figuières \& Grolleau, 2013; Osbaldiston \& Schott, 2012; Rubens, Gosling, Bonaiuto, Brisbois \& Moch, 2015; Obradovich \& Guenther, 2016; Rashid \& Mohammad, 2012); influência social (Tanford \& Montgomery, 2015; Beretti et al., 2013; Stenman \& Martinsson, 2006); feedback (Brook, 2011); informações sobre produtos sustentáveis (Bodur, Duval \& Grohmann, 2015; MacDonald \& She, 2015); negação e supressão de culpa (Cohen, Higham \& Cavaliere, 2011; Truelove, Yeung, Carrico, Gillis \& Raimi, 2016); compensação (Lavergne \& Pelletier, 2015; Cohen et al., 2011); justificação (Juvan \& Dolnicar, 2014; Kovács, Pantya, Medves, Hidegkuti, Heim \& Bursavich, 2014; Kimura \& Shinoki, 2007; Sottile, Meloni \& Cherchi, 2015); normas morais (Martinsson \& Lundqvist, 2010; Thogersen, 2004); benefícios percebidos versus custos/barreiras (Schultz, 2014; Tobler, Visschers \& Siegrist, 2012); e semelhança comportamental (Truelove, Carrico, Weber, Raimi \& Vandenbergh, 2014).

O aprofundamento desse tema é relevante, pois existe um grande interesse, principalmente dos governantes, em adotar políticas que contribuam para 0 desenvolvimento de estilos de vida mais amigáveis com o meio ambiente (Whitmarsh \& O’Neill, 2010). Em termos teóricos, Tanford e Montgomery (2015) destacam que há poucos estudos no século 21 que relacionam a dissonância cognitiva ao consumo sustentável. Nesse sentido, observa-se que há um vasto campo de pesquisa a ser explorado e aplicado ao comportamento do consumidor.

Ademais, a compreensão das mudanças de atitudes e comportamentos diante dos conflitos cognitivos relacionados ao consumo de recursos naturais pode, também, servir de suporte às empresas para formular ações de marketing sustentáveis e com maior valor agregado aos seus stakeholders. Permite, ainda, criar objetivos organizacionais alinhados à conservação do meio ambiente, influenciando o comportamento de consumo das pessoas, e, consequentemente, gerando resultados para a organização (Gordon, 2013; Peattie \& Peattie, 2009). Além disso, o entendimento desses conflitos cognitivos pode auxiliar as 
organizações no acompanhamento mais efetivo do cliente no processo de pósvenda, caso a compra tenha gerado alguma experiência negativa (Liang, 2016).

Este artigo está estruturado a partir das seções a seguir descritas. Na seção 2, apresentam-se o método utilizado e a forma de como foram coletados os dados. $\mathrm{Na}$ seção 3, discutem-se os resultados a partir da apresentação das características gerais dos artigos e da análise das variáveis. Por fim, na seção 4, apresentam-se as considerações finais deste artigo.

\section{Método}

A partir de uma revisão sistemática da literatura, buscou-se identificar e descrever as diferentes maneiras de como as pessoas lidam com a tensão gerada pela dissonância cognitiva em relação ao consumo sustentável. A revisão sistemática da literatura é uma metodologia confiável, rigorosa e auditável para validar e interpretar pesquisas prévias relacionadas a um fenômeno particular de interesse (Jalonen, 2012). Conforme Petticrew e Roberts (2008), uma revisão sistemática deve ser produzida quando uma imagem mais abrangente da evidência em um tópico da área é necessária para direcionar futuros esforços de pesquisa. Para tanto, foram considerados os critérios de inclusão dos artigos e a estratégia para localizar e selecioná-los, conforme descrito a seguir.

Tal revisão de literatura foi conduzida através de duas fases. Na primeira, foi realizada uma consulta na base de dados Web of Science que é definida como uma base multidisciplinar que indexa os periódicos mais citados em suas respectivas áreas. A opção por essa base justifica-se pela sua confiabilidade e ampla utilização entre os pesquisadores.

A pesquisa foi realizada aplicando-se o termo dissonância cognitiva (cognitive dissonance) com os seguintes termos: consumo sustentável (sustainable consumption), meio ambiente (environment), pró-ambiental (pro-environmental), atitudes pró-ambientais (pro-environmental atitudes), comportamento pró-ambiental (pro-environmental behavior), identidade ambiental (environmental identity), preocupação ambiental (environmental concern), desempenho ambiental (environmental performance), gestão ambiental (environmental management), atitudes ambientais (environmental atitudes), comportamento ambiental 
(environmental behavior), comportamento de conservação (behaviour conservation), comportamento ecológico (ecological behavior), sustentabilidade (sustainability), sustentável (sustainable), verde (green), consumo verde (green consumption) e consumo consciente (conscious consumption). Vale ressaltar que essa busca foi configurada para localizar estes termos no título, no resumo e nas palavras-chave, sem filtro por área de conhecimento e considerando-se todo o período de abrangência da base de dados que data de 1945 a 2016. Essa busca resultou em 128 artigos.

$\mathrm{Na}$ segunda fase, os resumos desses artigos foram lidos e foram, posteriormente, selecionados 22 artigos obedecendo-se os seguintes critérios de inclusão: (1) estar disponível na base de dados; (2) ser um estudo teórico ou empírico com foco na dissonância cognitiva relacionada ao consumo sustentável. Para tanto, assumiu-se, que

consumo sustentável é o uso de produtos e serviços que respondam às necessidades básicas e trazem uma melhor qualidade de vida, minimizando, ao mesmo tempo, o uso de recursos naturais, materiais tóxicos e emissões de resíduos e poluentes ao longo do ciclo de vida, de modo a não comprometer as necessidades das gerações futuras" (Simpósio de Oslo, 1994).

Seguem-se ainda outro critério relativo à escolha dos artigos, sendo: (3) ter os termos mencionados no título, no resumo e nas palavras-chave. Alguns artigos foram excluídos, pois um dos termos constava no título e outro no resumo, porém não estavam relacionados diretamente ao tema do presente estudo. Além disso, alguns foram excluídos pois estavam associados a outras áreas do conhecimento. Os artigos selecionados foram analisados qualitativamente e, na seção seguinte, são apresentados os resultados dessa análise.

\section{Resultados}

Antes da análise aprofundada dos artigos, ressalta-se que foram publicados poucos que relacionam o consumo sustentável com a teoria da dissonância cognitiva, conforme pode ser observado na Tabela 1. Nota-se, ainda, que as pesquisas relacionadas a esse tema ainda são muito recentes, evidenciando que este campo de pesquisa pode estar em fase de amadurecimento. Essa pode ser uma explicação plausível pelo baixo número de artigos publicados, mostrando, desse modo, que ainda há um vasto campo de pesquisa a ser explorado. Por outro 
lado, poder-se-ia se pensar, também, que pode haver pouco interesse dos pesquisadores em relação a esse tema. Porém, Thogersen (2004) mostra a relevância da temática ao afirmar que o desejo de evitar a inconsistência entre atitude e comportamento é um importante fator de formação de padrões de comportamento no campo do consumo sustentável. Enfim, determinar o nível de consistência comportamental é apoiado pelo desejo de evitar a dissonância cognitiva.

\section{Tabela 1}

Fator de impacto

\begin{tabular}{|c|c|c|c|c|}
\hline Autor & $\begin{array}{l}\text { Fator de } \\
\text { Impacto }\end{array}$ & Ano & $\begin{array}{l}\text { Total de } \\
\text { citações } \\
\text { (Google } \\
\text { Schollar) }\end{array}$ & $\begin{array}{l}\text { Média de } \\
\text { citações por } \\
\text { ano }\end{array}$ \\
\hline Beretti et al. & 133 & 2013 & 10 & 3,33 \\
\hline Obradovich e Guenther & 130 & 2016 & 3 & 3,00 \\
\hline Juvan e Dolnicar & 108 & 2014 & 37 & 18,50 \\
\hline Cohen et al. & 108 & 2011 & 80 & 16,00 \\
\hline Truelove et al. & 103 & 2014 & 46 & 23,00 \\
\hline Bodur et al. & 98 & 2015 & 2 & 2,00 \\
\hline MacDonald e She & 96 & 2015 & 12 & 12,00 \\
\hline Truelove et al. & 84 & 2016 & 2 & 2,00 \\
\hline Lavergne e Pelletier & 84 & 2015 & 3 & 3,00 \\
\hline Kovacz et al. & 84 & 2014 & 5 & 2,50 \\
\hline Osbaldiston e Schott & 84 & 2012 & 219 & 43,80 \\
\hline Tobler et al. & 84 & 2012 & 69 & 17,25 \\
\hline Thogersen & 84 & 2004 & 195 & 16,25 \\
\hline Stenman e Martinsson & 79 & 2006 & 195 & 16,25 \\
\hline Tanford e Montgomery & 78 & 2015 & 16 & 8,00 \\
\hline Rubens et al. & 78 & 2015 & 11 & 3,67 \\
\hline Martinsson e Lundqvist & 44 & 2010 & 31 & 5,17 \\
\hline Schultz & 35 & 2014 & 48 & 24,00 \\
\hline Rashid e Mohammad & 22 & 2012 & 5 & 1,25 \\
\hline Brook & 13 & 2011 & 15 & 3,00 \\
\hline Kimura e Shinoki & 5 & 2007 & 0 & 0,00 \\
\hline Sottile, Meloni e Cherchi & 0 & 2015 & 2 & 2,00 \\
\hline
\end{tabular}

Nota Fonte: Elaborada pelos autores (2016)

Tendo presente que existe uma lacuna entre atitude e comportamento no âmbito do consumo sustentável, descobriram-se diferentes mecanismos utilizados pelas pessoas para resolver essa inconsistência. A identificação das variáveis ocorreu a partir da leitura e da análise dos artigos considerando aspectos de 
semelhança que possibilitaram o seu agrupamento. A Tabela 2 apresenta os artigos selecionados em ordem alfabética, destacando-se os autores, o objetivo, a metodologia, a amostra e, por fim, os contextos em que os estudos foram realizados. Cada uma dessas variáveis é abordada nas subseções seguintes.

\section{Tabela 2}

Informações dos artigos selecionados

\begin{tabular}{|c|c|c|c|c|}
\hline Artigo & Objetivo do estudo & Metodologia & $\begin{array}{l}\text { Caracterização } \\
\text { da amostra }\end{array}$ & Variáveis \\
\hline $\begin{array}{l}\text { Beretti et al. } \\
(2013)\end{array}$ & $\begin{array}{l}\text { Mostrar que o esgotamento } \\
\text { dos recursos naturais pode } \\
\text { gerar a dissonância cognitiva } \\
\text { que, por sua vez, pode levar a } \\
\text { mudanças comportamentais a } \\
\text { fim de melhorar a relação com } \\
\text { o meio ambiente. }\end{array}$ & Teórico & Não aplicável & $\begin{array}{l}\text { - Comprometimento } \\
\text { - Influência social }\end{array}$ \\
\hline $\begin{array}{l}\text { Bodur et al. } \\
(2015)\end{array}$ & $\begin{array}{l}\text { Investigar se anúncios que } \\
\text { incluem um pedido de } \\
\text { consumo sustentável geram a } \\
\text { dissonância cognitiva e se } \\
\text { influenciam na preferência por } \\
\text { produtos ambientalmente } \\
\text { sustentáveis. }\end{array}$ & Empírico & $\begin{array}{l}101 \text { estudantes } \\
\text { de graduação }\end{array}$ & $\begin{array}{l}\text { - Informações } \\
\text { sobre produtos } \\
\text { sustentáveis }\end{array}$ \\
\hline $\begin{array}{l}\text { Brook } \\
(2011)\end{array}$ & $\begin{array}{l}\text { Examinar o efeito do feedback } \\
\text { da pegada ecológica no } \\
\text { comportamento pró-ambiental } \\
\text { e a sua relação com a } \\
\text { dissonância cognitiva. }\end{array}$ & Empírico & $\begin{array}{l}212 \text { estudantes } \\
\text { de graduação }\end{array}$ & - Feedback \\
\hline $\begin{array}{l}\text { Cohen et al. } \\
(2011)\end{array}$ & $\begin{array}{l}\text { Avaliar a proposição de que } \\
\text { excessivas viagens aéreas } \\
\text { turísticas podem constituir um } \\
\text { vício comportamental e como } \\
\text { os consumidores utilizam as } \\
\text { estratégias de supressão e } \\
\text { negação de culpa para reduzir } \\
\text { a dissonância cognitiva. }\end{array}$ & Empírico & $\begin{array}{l}30 \\
\text { consumidores } \\
\text { da Noruega e } \\
\text { do Reino Unido }\end{array}$ & $\begin{array}{l}\text { - Negação e } \\
\text { supressão de culpa } \\
\text { - Compensação }\end{array}$ \\
\hline $\begin{array}{l}\text { Juvan e } \\
\text { Dolnicar } \\
(2014)\end{array}$ & $\begin{array}{l}\text { Investigar por que as pessoas } \\
\text { que se envolvem ativamente } \\
\text { na proteção do meio ambiente } \\
\text { em casa se envolvem em } \\
\text { comportamentos de férias que } \\
\text { têm consequências } \\
\text { ambientais negativas e como } \\
\text { elas resolvem essa lacuna } \\
\text { entre atitude e } \\
\text { comportamento. }\end{array}$ & Empírico & $\begin{array}{l}25 \text { membros de } \\
\text { organizações } \\
\text { ambientais da } \\
\text { Austrália e } \\
\text { Eslovênia }\end{array}$ & - Justificação \\
\hline
\end{tabular}




\begin{tabular}{|c|c|c|c|c|}
\hline $\begin{array}{l}\text { Kimura e } \\
\text { Shinoki } \\
\text { (2007) }\end{array}$ & $\begin{array}{l}\text { Construir um modelo de dois } \\
\text { estágios abordando a tomada } \\
\text { de decisão e a utilização de } \\
\text { estratégias de justificação } \\
\text { para reduzir a dissonância } \\
\text { cognitiva em uma situação } \\
\text { potencial de dilema social, } \\
\text { focando o problema da } \\
\text { reciclagem. }\end{array}$ & Teórico & Não aplicável & - Justificação \\
\hline $\begin{array}{l}\text { Kovács et } \\
\text { al. (2014) }\end{array}$ & $\begin{array}{l}\text { Analisar o papel da } \\
\text { justificação diante de um } \\
\text { comportamento } \\
\text { ambientalmente prejudicial e } \\
\text { a sua relação com a } \\
\text { dissonância cognitiva. }\end{array}$ & Empírico & $\begin{array}{l}200 \text { cidadãos } \\
\text { americanos e } \\
\text { húngaros }\end{array}$ & - Justificação \\
\hline $\begin{array}{l}\text { Lavergne e } \\
\text { Pelletier } \\
\text { (2015) }\end{array}$ & $\begin{array}{l}\text { Examinar como as pessoas } \\
\text { resolvem as inconsistências } \\
\text { entre as suas atitudes pró- } \\
\text { ambientais e suas ações não } \\
\text { pró-ambientais a partir da } \\
\text { utilização de estratégias para } \\
\text { compensar e resolver esse } \\
\text { conflito cognitivo. }\end{array}$ & Empírico & $\begin{array}{l}\text { Estudo 1: } 429 \\
\text { estudantes } \\
\text { Estudo 2: } 257 \\
\text { estudantes }\end{array}$ & - Compensação \\
\hline $\begin{array}{l}\text { MacDonald } \\
\text { e She } \\
\text { (2015) }\end{array}$ & $\begin{array}{l}\text { Realizar uma revisão da } \\
\text { pesquisa para identificar } \\
\text { maneiras de como incentivar } \\
\text { o comportamento pró- } \\
\text { ambiental e o consumo de } \\
\text { produtos ecológicos. }\end{array}$ & Teórico & Não aplicável & $\begin{array}{l}\text { - Informações } \\
\text { sobre produtos } \\
\text { sustentáveis }\end{array}$ \\
\hline $\begin{array}{l}\text { Martinsson } \\
\text { e Lundqvist } \\
\text { (2010) }\end{array}$ & $\begin{array}{l}\text { Examinar se os cidadãos que } \\
\text { possuem valores e atitudes } \\
\text { compatíveis com o modelo de } \\
\text { cidadania ecológica exibem } \\
\text { tais práticas verdes e agem } \\
\text { de acordo com suas crenças } \\
\text { e atitudes em relação ao meio } \\
\text { ambiente. }\end{array}$ & Empírico & $\begin{array}{l}\text { Relatório de } \\
\text { pesquisa } \\
\text { realizado com } \\
\text { cidadãos da } \\
\text { Suécia }\end{array}$ & - Normas morais \\
\hline $\begin{array}{l}\text { Obradovich } \\
\text { e Guenther } \\
\text { (2016) }\end{array}$ & $\begin{array}{l}\text { Investigar a eficiência da } \\
\text { responsabilidade individual e } \\
\text { coletiva relacionadas às } \\
\text { mudanças climáticas e a } \\
\text { relação com a dissonância } \\
\text { cognitiva. }\end{array}$ & Empírico & 1.200 pessoas & - Comprometimento \\
\hline $\begin{array}{l}\text { Osbaldiston } \\
\text { e Schott } \\
\text { (2012) }\end{array}$ & $\begin{array}{l}\text { Fornecer para os } \\
\text { investigadores, profissionais e } \\
\text { ambientalistas informações } \\
\text { necessárias para projetar } \\
\text { intervenções e promover } \\
\text { comportamentos pró- } \\
\text { ambientais eficazes. }\end{array}$ & Empírico & 87 relatórios & - Comprometimento \\
\hline
\end{tabular}




\begin{tabular}{|c|c|c|c|c|}
\hline $\begin{array}{l}\text { Rashid e } \\
\text { Mohammad } \\
\text { (2012) }\end{array}$ & $\begin{array}{l}\text { Explicar através da teoria da } \\
\text { dissonância cognitiva o } \\
\text { processo ou estágios do } \\
\text { fenômeno de envolvimento } \\
\text { das pessoas em um } \\
\text { comportamento pró- } \\
\text { ambiental. }\end{array}$ & Teórico & Não aplicável & - Comprometimento \\
\hline $\begin{array}{l}\text { Rubens et } \\
\text { al. (2015) }\end{array}$ & $\begin{array}{l}\text { Explorar o efeito de dois tipos } \\
\text { de intervenções que são } \\
\text { usados para modificar } \\
\text { comportamentos pró- } \\
\text { ambientais: compromisso e o } \\
\text { paradigma hipocrisia (com } \\
\text { dissonância cognitiva). }\end{array}$ & Empírico & $\begin{array}{l}74 \text { clientes de } \\
\text { um } \\
\text { supermercado } \\
\text { em Paris }\end{array}$ & - Comprometimento \\
\hline $\begin{array}{l}\text { Schultz } \\
(2014)\end{array}$ & $\begin{array}{l}\text { Sintetizar os resultados da } \\
\text { investigação sobre as várias } \\
\text { estratégias, entre elas a } \\
\text { dissonância cognitiva, } \\
\text { utilizadas para promover } \\
\text { comportamentos pró- } \\
\text { ambientais e examinar as } \\
\text { condições que maximizam a } \\
\text { eficácia de cada um. }\end{array}$ & Teórico & Não aplicável & $\begin{array}{l}\text { - Benefícios } \\
\text { percebidos versus } \\
\text { custos/barreiras }\end{array}$ \\
\hline $\begin{array}{l}\text { Sottile et al. } \\
(2015)\end{array}$ & $\begin{array}{l}\text { Compreender a relação entre } \\
\text { consciência, a discrepância } \\
\text { entre atitude e } \\
\text { comportamento no contexto } \\
\text { do transporte ecológico. }\end{array}$ & Empírico & 85 participantes & - Justificação \\
\hline $\begin{array}{l}\text { Stenman e } \\
\text { Martinsson } \\
\text { (2006) }\end{array}$ & $\begin{array}{l}\text { Analisar como o status e a } \\
\text { preocupação com o meio } \\
\text { ambiente interferem na } \\
\text { decisão de compra de um } \\
\text { carro e a influência da } \\
\text { dissonância cognitiva nessa } \\
\text { decisão. }\end{array}$ & Empírico & $\begin{array}{l}1.300 \\
\text { participantes }\end{array}$ & - Influência social \\
\hline $\begin{array}{l}\text { Tanford e } \\
\text { Montgomery } \\
\text { (2015) }\end{array}$ & $\begin{array}{l}\text { Avaliar o impacto da } \\
\text { influência social e atitudes } \\
\text { ambientais através da } \\
\text { dissonância ao escolher entre } \\
\text { um resort sustentável e um } \\
\text { resort não sustentável. } \\
\text { Avaliar os consumidores na } \\
\text { medida em que se envolvem } \\
\text { em estratégias de redução da } \\
\text { dissonância depois de } \\
\text { fazerem uma escolha. }\end{array}$ & Empírico & $\begin{array}{l}308 \text { estudantes } \\
\text { de graduação }\end{array}$ & - Influência social \\
\hline $\begin{array}{l}\text { Thogersen } \\
\text { (2004) }\end{array}$ & $\begin{array}{l}\text { Analisar se os } \\
\text { comportamentos } \\
\text { ambientalmente sustentáveis } \\
\text { são positivamente } \\
\text { correlacionados à luz da } \\
\text { teoria da dissonância } \\
\text { cognitiva. }\end{array}$ & Empírico & $\begin{array}{l}309 \\
\text { consumidores } \\
\text { dinamarqueses }\end{array}$ & - Normas morais \\
\hline
\end{tabular}


(conclusão)

\begin{tabular}{|c|c|c|c|c|}
\hline $\begin{array}{l}\text { Tobler et al. } \\
\text { (2012) }\end{array}$ & $\begin{array}{l}\text { Encontrar uma forma } \\
\text { significativa para classificar } \\
\text { diferentes formas de lidar com } \\
\text { a mudança climática. } \\
\text { Analisar os fatores } \\
\text { determinantes que } \\
\text { influenciam a disposição das } \\
\text { pessoas para adotar } \\
\text { comportamentos sustentáveis } \\
\text { diante de um conflito } \\
\text { cognitivo. }\end{array}$ & Empírico & 916 pessoas & $\begin{array}{l}\text { - Benefícios } \\
\text { percebidos versus } \\
\text { custos/barreiras }\end{array}$ \\
\hline $\begin{array}{l}\text { Truelove et } \\
\text { al. (2014) }\end{array}$ & $\begin{array}{l}\text { Realizar uma avaliação } \\
\text { interdisciplinar para } \\
\text { esclarecer as condições em } \\
\text { que um efeito positivo ou } \\
\text { negativo pode ser esperado } \\
\text { diante de comportamentos } \\
\text { pró-ambientais. }\end{array}$ & Teórico & Não aplicável & $\begin{array}{l}\text { - Semelhança } \\
\text { comportamental }\end{array}$ \\
\hline $\begin{array}{l}\text { Truelove et } \\
\text { al. (2016) }\end{array}$ & $\begin{array}{l}\text { Examinar simultaneamente a } \\
\text { capacidade de identidade } \\
\text { ambiental, culpa e } \\
\text { preocupação para explicar os } \\
\text { efeitos de um comportamento } \\
\text { pró-ambiental. }\end{array}$ & Empírico & $\begin{array}{l}283 \text { estudantes } \\
\text { universitários } \\
\text { dos Estados } \\
\text { Unidos }\end{array}$ & $\begin{array}{l}\text { - Negação e } \\
\text { supressão de culpa }\end{array}$ \\
\hline
\end{tabular}

Nota Fonte: Elaborado pelos autores (2016)

\subsection{Comprometimento}

As pessoas se comprometem, com antecedência, a um determinado comportamento pró-ambiental quando, publicamente, assumem um compromisso. Além disso, induzir e fazer com que se comprometam com pequenos comportamentos pode levar a uma oportunidade para um comportamento maior e até mais relevante. As estratégias de compromisso também podem ser eficazes quando os benefícios e as barreiras são elevados.

Inovações comportamentais podem incentivar as pessoas a fazerem melhores escolhas e podem, portanto, constituir poderosas soluções para as questões de sustentabilidade (Beretti et al., 2013). Conforme os autores, fazer com que as pessoas se comprometam com antecedência para um determinado comportamento, de preferência publicamente, pode aumentar a adoção de um comportamento próambiental e, para evitar a dissonância cognitiva, este será condizente com a sua atitude. 
Nesse mesmo sentido, Obradovich e Guenther (2016) realizaram três experimentos e concluíram que há várias razões pelas quais a responsabilidade coletiva produz respostas mais positivas em relação a comportamentos próambientais para mitigar as mudanças climáticas do que a responsabilidade individual, sendo que uma dessas razões é evitar a dissonância cognitiva.

Considerando que as pessoas têm a possibilidade de adotar comportamentos pró-ambientais, Osbaldiston e Schott (2012) analisaram 87 relatórios publicados entre 1980 e 2009 que resultaram em um total de 253 tratamentos. Desses, foram selecionados 10 tipos de tratamentos, sendo que um deles é a dissonância cognitiva relacionada aos processos sócio psicológicos. Osbaldiston e Schott (2012) destacam que a dissonância cognitiva permite acessar crenças ou atitudes preexistentes e faz os participantes se comportarem de maneira coerente para reduzir o desconforto psicológico. Nesses tratamentos, notou-se que os participantes se envolvem e se comprometem, primeiramente, em um pequeno comportamento e, posteriormente, em um comportamento maior.

Rubens et al. (2015) realizaram um experimento em um supermercado de Paris relacionado à utilização de sacos plásticos. A finalidade foi de verificar a hipótese de que o atraso entre a intervenção e o momento em que ocorre o comportamento permitiria a redução de dissonância via justificativas defensivas e que os participantes na condição de compromisso (que não estavam vivenciando dissonância) iriam mostrar mais comportamentos e atitudes consistentes do que os participantes na condição de hipocrisia (que estavam experimentando dissonância). Em relação à condição de compromisso, os autores concluíram que os participantes que se comprometeram a assinar um pôster defendendo o comportamento próambiental, eram mais propensos a mudar seu comportamento do que os participantes do grupo de controle. Além disso, os resultados mostraram que os participantes que se comprometeram no início de suas compras, adotaram o comportamento pró-ambiental no final de suas compras, quando optaram em não utilizar os sacos. Os participantes, na condição de hipocrisia, não adotaram comportamentos pró-ambientais e mesmo que a dissonância cognitiva tenha sido despertada, eles continuaram fazendo suas compras e tiveram tempo para banalizar ou racionalizar o seu comportamento.

Em seu estudo, Rashid e Mohammad (2012), afirmam que os indivíduos são influenciados pelo ambiente em que vivem quando se trata de um comportamento 
amigável com o meio ambiente. Quando se comprometem com práticas de consumo sustentável na empresa em que trabalham, os indivíduos tendem a ter comportamentos pró-ambientais na sociedade e também na sua casa porque desejam evitar a sensação de desconforto gerada pela dissonância cognitiva, caso não ajam de acordo com as suas atitudes e comportamentos anteriormente assumidos.

\subsection{Influência social}

Os comentários e as opiniões influenciam a decisão de adotar ou não comportamentos sustentáveis, considerando que a maioria das pessoas buscam ser socialmente responsáveis.

Tanford e Montgomery (2015) realizaram uma pesquisa com estudantes de graduação para verificar o comportamento diante da escolha entre um resort verde e um não verde para um período de férias. $O$ estudo concluiu que a dissonância cognitiva e a influência social desempenham um papel importante nas decisões de compra de uma viagem. Os resorts verdes são considerados atraentes por todos, enquanto os resorts não verdes estão em desvantagem, quando se trata de convidados com comportamentos pró-ambientais. Segundo observado pelos autores, depois de tomar a decisão, os indivíduos experimentaram a dissonância. Como previsto, a dissonância foi maior na condição de influência minoritária, pois uma escolha não verde produziu maior desconforto do comprador, quando havia um único comentário favorável para o resort verde. Sendo assim, considera-se que as cognições dissonantes motivam as pessoas a buscar informações agradáveis e evitar informações não agradáveis. Mesmo se uma maioria dos comentários é favorável, um único comentário negativo pode ser determinante na escolha. Esta descoberta sugere que os clientes desejam ser socialmente responsáveis, fazendo escolhas verdes, mas precisam de informações para justificá-las (Tanford \& Montgomery, 2015).

Beretti et al. (2013) apoiam esses resultados, ao afirmar que a influência social pode ser usada para promover o consumo sustentável, por descrever o comportamento desejado como uma norma social descritiva quando isso for possível, ou como uma norma prescritiva moral, nos outros casos. Da mesma forma, 
o status pode ser aproveitado para promover o consumo sustentável, facilitando comparações entre entidades, com base em desempenhos ambientalmente amigáveis.

Corroborando os estudos acima citados, Stenman e Martinsson (2006) realizaram uma pesquisa com consumidores da Suécia para verificar os atributos considerados mais importantes na tomada de decisão da compra de um carro. Os autores concluíram que as pessoas tendem a ser mais preocupadas com o status e menos preocupadas com o meio ambiente quando estão prestes a comprar um veículo. Porém, como culturalmente a preocupação com o status é percebida como um mal social e a preocupação ambiental um bem social, as pessoas tentam mostrar para a sociedade de que são muito mais preocupadas com as questões de sustentabilidade. O desejo de evitar a dissonância cognitiva faz com que as pessoas alterem sua atitude para convencer e mostrar aos outros a sua preocupação.

\subsection{Feedback}

No que diz respeito ao feedback, pode-se afirmar que ele pode aumentar ou até mesmo diminuir o comportamento ambientalmente sustentável. Essa variabilidade é, muitas vezes, influenciada pela autoestima das pessoas em ter comportamentos pró-ambientais.

Brook (2011) realizou um experimento com estudantes, a fim de examinar o efeito do feedback da pegada ecológica no comportamento pró-ambiental e se esse efeito depende da autoestima das pessoas. Os resultados evidenciaram que os participantes que basearam a sua autoestima no ambientalismo eram mais propensos a se envolver em comportamentos pró-ambientais após o feedback da pegada ecológica negativo do que os participantes que não basearam a sua autoestima no ambientalismo. Enfim, o feedback da pegada ecológica pode aumentar ou diminuir o comportamento ambientalmente sustentável, dependendo do quanto as pessoas estão comprometidas com o ambientalismo através da sua autoestima. Além disso, Brook (2011) ainda destaca que as pessoas que possuem comportamentos pró-ambientais são mais propensas a resolver a dissonância cognitiva, alterando seu comportamento para corresponder às suas atitudes, em vez de adotar outros meios para reduzir a dissonância. Alguns participantes foram menos propensos a se envolver em um comportamento pró-ambiental após o 
feedback negativo, presumivelmente porque não experimentaram a dissonância. Embora possa induzir comportamentos pró-ambientais entre as pessoas que já estão altamente comprometidos com o ambientalismo, pode, ao mesmo tempo, prejudicar o comportamento das pessoas que ainda não estão comprometidas.

\subsection{Informações sobre produtos sustentáveis}

As informações em alguns produtos, destacando o impacto ambiental, podem aumentar ou diminuir a preferência das pessoas por itens sustentáveis. Além disso, os pedidos e as mensagens de consumo sustentável também podem interferir significativamente.

Em um estudo realizado com estudantes canadenses para verificar a preferência por produtos ecológicos e não ecológicos, Bodur et al. (2015) demonstraram a eficácia do uso de pedidos de consumo sustentável em anúncios para aumentar a preferência por produtos ambientalmente sustentáveis. Propagandas que provocam um compromisso de comprar produtos sustentáveis, através da inclusão de informações sobre o impacto ambiental, levaram a um aumento da preferência por itens sustentáveis, em comparação com um controle de anúncio que incluiu uma simples mensagem que se referia à compra de tais produtos. Ao mesmo tempo, mostrou que a suscetibilidade aos efeitos de um pedido de consumo sustentável é maior para os indivíduos que são mais interdependentes, particularmente quando o anúncio também inclui uma sugestão pública. Conforme Bodur et al. (2015), para os indivíduos interdependentes, aumentar a natureza pública de uma escolha evoca mais fortemente a dissonância cognitiva, que conduz a um maior esforço para tentar reduzi-la.

Por outro lado, MacDonald e She (2015), a partir de uma revisão de literatura, concluíram que antes de introduzir um novo produto ecológico, é importante estudar as impressões dos clientes sobre o impacto das ofertas dos produtos atuais. A introdução de um item ecológico pode gerar nos clientes a dissonância cognitiva, pois a mesma empresa também disponibiliza outro produto que ele já compra atualmente e que não possui esse caráter ecológico. Essa situação pode ser evitada posicionando os dois produtos para que não sejam comparados diretamente. 
Conforme MacDonald e She (2015), deve-se evitar que os clientes se sintam culpados ou experimentem a dissonância cognitiva.

\subsection{Negação e supressão de culpa}

A fim de justificar os benefícios pessoais das ações que ocasionam mudanças climáticas e prejudicam o meio ambiente, algumas pessoas, para evitar a dissonância cognitiva, utilizam estratégias de negação e supressão de culpa.

Cohen et al. (2011) realizaram um estudo sobre o comportamento dos consumidores da Noruega e do Reino Unido relacionado a viagens aéreas e as consequentes alterações climáticas. Os resultados confirmaram que vários participantes expressaram preocupação com as consequências climáticas, mas utilizavam muito o transporte aéreo para viagens turísticas de curtas distâncias e não estavam dispostos a abandonar as suas experiências de turismo. Para evitar a dissonância cognitiva e para não mudar o seu comportamento, utilizaram estratégias de negação e supressão de culpa para justificar os benefícios pessoais de curto prazo do turismo, associadas às consequências de longo prazo das mudanças climáticas.

Truelove et al. (2016) realizaram uma pesquisa com estudantes universitários dos Estados Unidos a fim de investigar a reação dos discentes em relação à reciclagem de uma garrafa de água. Conforme os autores, nesse estudo encontraram-se poucas evidências de que a identidade ambiental, a culpa e a preocupação com o meio ambiente medissem a relação entre a reciclagem e o apoio a uma política ambiental. Enfim, pressupõe-se que os indivíduos não sentiram desconforto ou culpa por não terem adotado algum comportamento pró-ambiental e apoiado políticas públicas pró-ambientais.

\subsection{Compensação}

As estratégias de compensação são utilizadas pelas pessoas para minimizar as consequências aversivas de suas ações. A motivação controlada provoca atitudes pró-ambientais enfraquecidas, desencadeando em inconsistências mais frequentes. Por outro lado, a motivação autônoma leva a fortes atitudes próambientais e inconsistências menos frequentes. 
Lavergne e Pelletier (2015) afirmam que as pessoas consideram a proteção ambiental importante e se envolvem em comportamentos pró-ambientais por diferentes razões e essas determinam o significado do uso de estratégias de compensação. A motivação controlada dispõe pessoas para monitorar e compensar suas ações prejudiciais ao ambiente somente quando essas ações têm o potencial de gerar consequências aversivas. Quando as ações não geram consequências potencialmente aversivas, elas parecem inclinadas a mudar ou banalizar suas atitudes pró-ambientais para evitar a dissonância, mas quando geram consequências potencialmente aversivas, despertam a dissonância e motivam as pessoas a compensar através do uso de estratégias para modificar o comportamento, a fim de minimizar as consequências aversivas de suas ações. Os resultados do estudo sugerem que os processos de compensação, associados à motivação autônoma, são direcionados a resolver a inconsistência, proteger o meio ambiente, em vez de apenas reduzir a dissonância. Já os processos de compensação associados a altos níveis de motivação controlada podem levar a atitudes pró-ambientais enfraquecidas, inconsistências mais frequentes e redução da motivação para o meio ambiente. Para Lavergne e Pelletier (2015), encontrar formas para promover a motivação autônoma durante os processos de compensação pode potencialmente reduzir a lacuna entre atitude e comportamento. A motivação controlada gera a dissonância cognitiva, pois as pessoas são pressionadas a ter comportamentos pró-ambientais quando, na verdade, possuem fracas atitudes próambientais.

Um dos resultados do estudo de Cohen et al. (2011) sobre o comportamento dos consumidores da Noruega e no Reino Unido relacionado com viagens aéreas, e consequentes alterações climáticas, também evidenciou o uso de estratégias de compensação para reduzir a lacuna provocada pela dissonância cognitiva.

\subsection{Justificação}

Para evitar a dissonância cognitiva, as pessoas utilizam diferentes justificativas, a seguir destacadas: "não é tão ruim assim"; "poderia ser pior"; "não é minha responsabilidade"; "eu gostaria, mas..."; "as férias são uma exceção"; "na verdade, eu estou fazendo mais bem do que mal". Além disso, destaca-se que a 
utilização de justificativas varia conforme a cultura e também devido a fatores externos.

Juvan e Dolnicar (2014) realizaram entrevistas com membros de organizações ambientais da Austrália e da Eslovénia que eram altamente conscientes das consequências ambientais negativas do turismo, mas todos exibiram uma lacuna entre atitude e comportamento que gerou um desconforto. Para evitar a dissonância cognitiva, os participantes ofereceram uma ampla gama de explicações para justificar as suas atividades turísticas. A partir dessas explicações, Juvan e Dolnicar (2014) destacaram seis grupos de crenças: (1) "Não é tão ruim assim" - consistiu na negação das consequências negativas e os participantes não estavam com falta de consciência dos impactos ambientais negativos do turismo; (2) "Poderia ser pior" indicam que o comportamento de uma pessoa é contrastado com o comportamento de outras pessoas ou o impacto de indústrias; (3) "Não é minha responsabilidade" abrange os aspectos de uma verdadeira externalização da responsabilidade e, a partir da sensação de que o próprio comportamento não pode realmente fazer a diferença; (4) "Eu gostaria, mas..." - as pessoas argumentam que o comportamento não está sob seu controle e justificam afirmando que o turismo que não causa tanto impacto é muito caro e que não possuem tempo suficiente para fazer todas as investigações que seriam necessárias a fim de determinar qual opção de férias tem o menor impacto ambiental; (5) "As férias são uma exceção" - o comportamento de férias é uma exceção; e (6) "Na verdade, eu estou fazendo mais bem do que mal" reconhece o impacto negativo do turismo, mas encontra alinhamento com outras atitudes de preservação. Conforme Juvan e Dolnicar (2014), as crenças são desenvolvidas e modificadas ao longo da vida de uma pessoa e, portanto, representam um alvo mais realista para causar a mudança de comportamentos.

Da mesma forma, Kovács et al. (2014) realizaram uma pesquisa com cidadãos americanos e húngaros, com o intuito de analisar o papel da justificação do não comportamento como um fator inibidor, e de atitudes ambientais positivas, como um fator que encoraja um comportamento pró-ambiental. Os resultados reforçaram a ideia de que atitudes ambientais positivas encorajam um comportamento próambiental e, também, melhoram a criticidade percebida. Além disso, os sentimentos de criticidade percebida diminuem a aceitação da justificação dos comportamentos antagonistas e de que a cultura pode impedir um indivíduo de sentir cognições contraditórias, ao escolher um comportamento que não seja sustentável. De acordo 
com o estudo, a justificação depende da cultura. Enquanto que, entre os cidadãos americanos, a justificação teve uma influência direta e negativa sobre o comportamento ambiental, entre os húngaros não ocorreu essa influência. Os indivíduos podem justificar o não comportamento mesmo na presença de atitudes pró-ambientais, caso tenham uma tendência em escolher a opção prejudicial. De acordo com o modelo da dissonância cognitiva baseada em ação, uma ação orientada fornece uma base para a implementação de um comportamento eficiente, uma vez que implica a necessidade de cognições não conflitantes. $O$ apoio à implementação de um comportamento, reduzindo dissonância cognitiva, pode ser mais típico em americanos do que em húngaros.

Kimura e Shinoki (2007) propõem um modelo de duas etapas relacionado a problemas de reciclagem: tomada de decisão e estratégias de justificação para reduzir a dissonância cognitiva. Na primeira etapa, os indivíduos tomam a decisão de fazer ou não a reciclagem. Já na segunda etapa, alguns indivíduos podem deixar de implementar suas decisões por diversos fatores externos, surgindo assim a dissonância cognitiva. Esses indivíduos são motivados a reduzi-la justificando as suas ações de três maneiras: mudam a cognição sobre a probabilidade de que o sistema de reciclagem funciona; alteram a cognição sobre o valor do bom funcionamento do sistema de reciclagem; alteram a cognição no custo para a contribuição para a reciclagem.

Sottile et al. (2015) concluíram em seu estudo que os usuários de automóveis muitas vezes acham difícil mudar seu comportamento de viagem usando modos mais sustentáveis de transporte. Apesar de estar ciente e bem informado de que um comportamento mais responsável é necessário para proteger o ambiente, isso não tem repercussões imediatas sobre o seu comportamento de viagem. Mesmo os usuários conscientes do meio ambiente que têm o cuidado de adotar um estilo de vida verde acham difícil e muitas vezes não conseguem se comprometer quando se trata de mudar seu comportamento de viagem. As justificativas para evitar a dissonância cognitiva, nesse caso, são a percepção de que o transporte público é pouco integrado e que o estilo de vida frenético impossibilita utilizar outro tipo de transporte que não seja o carro particular. Os autores apontam que medidas suaves para sensibilizar as pessoas para as consequências de um comportamento pouco sustentável não são suficientes para superar a dissonância cognitiva. 


\subsection{Normas morais}

O nível de normas morais interfere diretamente na decisão das pessoas em adotar comportamentos sustentáveis. Caso esse nível seja fraco, elas tendem a não se importar com as incoerências no seu comportamento.

Martinsson e Lundqvist (2010) acreditam que a questão central, quando se estuda a cidadania ecológica, é a correspondência entre as atitudes e os comportamentos dos indivíduos. A partir de um estudo realizado na Suécia, os autores definiram quatro categorias de indivíduos, em termos de atitudes ambientais relacionadas com comportamentos pró-ambientais: believers - indivíduos que mantêm atitudes positivas com o meio ambiente e apresentam comportamentos ecologicamente responsáveis; diehards - demonstram compromisso com o meio ambiente e apresentam coerência entre suas atitudes e seus comportamentos; hypocrites - expressam atitudes ambientais positivas e mostram preocupação ambiental, porém não traduzem essa preocupação em uma prática verde; e coverts indivíduos não comprometidos e com atitudes negativas que exibem práticas ecologicamente benignas. Nota-se que nas duas últimas categorias, os indivíduos apresentam incoerência entre as suas atitudes e os seus comportamentos. Diante disso, pode-se esperar uma tensão no caso dos hypocrites, o que configura a dissonância cognitiva. Para os indivíduos que não têm ou têm um fraco nível de normas morais, relacionadas ao comportamento com o meio ambiente, pouco importa se eles percebem incoerência em seu comportamento. No entanto, para os indivíduos com fortes normas morais, o desejo de evitar a dissonância cognitiva, cria neles uma unidade para se comportarem de forma coerente (Martinsson \& Lundqvist, 2010). O comportamento verde dos coverts não é acompanhado por quaisquer atitudes ambientais e nem por uma perspectiva moral. O comportamento cinza dos hypocrites se torna ainda mais dissonante quando considera que suas atitudes verdes estão intimamente ligadas a um olhar fortemente voltado para a cidadania ecológica.

Em um estudo realizado com consumidores dinamarqueses, Thogersen (2004) concluiu que os comportamentos ambientalmente sustentáveis são positivamente correlacionados, exceto nos casos em que os comportamentos são influenciados por condições pessoais. O autor concluiu que o desejo de evitar a inconsistência é de fato um importante fator na formação de padrões de comportamento no campo 
ambiental e que a interação entre normas morais e percepções de similaridade para determinar o nível de consistência comportamental podem conduzir este processo.

\subsection{Benefícios percebidos versus custos/barreiras}

Os consumidores tomam suas decisões, em relação ao consumo sustentável, sempre considerando os custos e os benefícios percebidos. No caso em que há altos benefícios e baixas barreiras, sugere-se a indução da dissonância cognitiva com o propósito de levar as pessoas a alterarem suas cognições ou seus comportamentos.

Para Schultz (2014), os problemas ambientais têm suas origens no comportamento humano e, como resultado, qualquer solução para as questões ambientais exigirá mudanças no comportamento. Para isso, o autor apresenta algumas ferramentas capazes de alterar o comportamento dos indivíduos, a partir da análise do quadro do Marketing Social Baseado na Comunidade, que tem como um dos seus principais componentes a ênfase em programas de desenvolvimento que diminuem diretamente barreiras e aumentam os benefícios associados com o comportamento alvo. Nesse sentido, Schultz (2014) propõe algumas ferramentas considerando algumas situações. Entre elas, na situação que envolve altos benefícios e baixas barreiras, o comportamento alvo é relativamente fácil e o público é motivado. Nesse caso, entre as ferramentas sugeridas, destaca-se a dissonância cognitiva. Uma vez induzida, a pessoa está motivada para reduzir a dissonância e alterar suas cognições ou seus comportamentos. Envolve a indução de pequenos comportamentos, apontando que esses refletem suas atitudes favoráveis em relação ao problema. Ademais, também induzem a terem um comportamento levando a uma oportunidade para um comportamento maior. Schultz (2014) ressalta que a dissonância é mais eficaz para um público-alvo que já está motivado a se engajar no comportamento.

Numa pesquisa realizada na Suíça, Tobler et al. (2012) relacionaram o comportamento das pessoas às mudanças climáticas e concluíram que os consumidores tomam suas decisões em relação a comportamentos pró-ambientais sempre considerando os custos e os benefícios percebidos. As pessoas tendem a superestimar suas contribuições para a mitigação das alterações climáticas e a 
subestimar o impacto negativo de suas ações. A forte influência de benefícios climáticos percebidos no estudo pode refletir uma estratégia para a redução da dissonância cognitiva. Como os comportamentos de alto custo são mais difíceis de adotar, os consumidores podem estar dispostos a mudar seu estilo de vida e, portanto, experimentam uma tensão desconfortável. Para reduzir a dissonância cognitiva, os consumidores podem descartar comportamentos de alto custo, considerando-os não efetivos e adotar comportamentos de baixo custo favoráveis ao clima e também apoiar as políticas de baixo custo.

\subsection{Semelhança comportamental}

No que diz respeito à semelhança comportamental, pode-se afirmar que, para evitar a dissonância cognitiva, as pessoas adotam comportamentos pró-ambientais semelhantes.

Truelove et al. (2014), a partir de uma revisão de literatura, identificaram diferentes modos de decisão como mecanismos que impulsionam a adoção de comportamentos pró-ambientais iniciais, com consequências diferentes para comportamentos pró-ambientais subsequentes, levando a um efeito positivo, negativo ou até mesmo a nenhum efeito. Os autores preveem que um efeito positivo pode ser mais provável entre dois comportamentos pró-ambientais semelhantes do que entre comportamentos pró-ambientais diferentes o que pode justificar o desejo de evitar a dissonância cognitiva. Por exemplo, alguém que recicla e deseja manter uma autoimagem de ser consistente, será mais propenso a se envolver em outros comportamentos semelhantes à reciclagem.

\section{Considerações Finais}

Até o presente estudo, considerava-se, de uma forma geral, que para evitar a tensão gerada pela dissonância cognitiva em relação ao consumo sustentável, as pessoas mudavam o seu comportamento, a sua atitude ou utilizavam estratégias de justificação. Porém, pode-se lidar de diferentes maneiras com esse desconforto psicológico entre a atitude e o comportamento de consumo. Assim, o presente estudo aponta para essas variáveis e incorpora na literatura pontos-chave para a geração de novas teorias psicológicas, bem como proporciona novas perspectivas 
para a solução de desafios da sustentabilidade no campo do comportamento de consumo. Pesquisas futuras utilizando essas variáveis como função do impacto direto ou indireto do consumo podem contribuir para o entendimento do ciclo de vida de produtos ou serviços sustentáveis e auxiliar na mitigação dos danos causados ao meio ambiente.

Diante disso, este estudo permite concluir, a partir da revisão sistemática da literatura, que existem algumas variáveis pelas quais as pessoas lidam com a lacuna existente entre atitude e comportamento: comprometimento, influência social, feedback, informações sobre produtos sustentáveis, negação e supressão de culpa, compensação, justificação, normas morais, benefícios percebidos versus custos/barreiras e semelhança comportamental. A identificação e a descrição dessas variáveis aprofundam o entendimento de que substituir, esquecer ou adquirir novas cognições resolvem a dissonância e especificam detalhadamente um conjunto de justificativas presentes no comportamento do consumidor ao lidar com o fenômeno.

Em relação às implicações teóricas, observa-se que, embora este artigo tenha identificado essas diferentes variáveis, cabe ressaltar que elas foram subtraídas de estudos realizados em diferentes contextos. Conclui-se, diante desse aspecto, que a maneira como as pessoas lidam com a tensão varia conforme a situação em que estão inseridas. Supõe-se também que, além dessas variáveis, ainda possam existir outras que interferem no consumo sustentável. Portanto, a identificação destas e a sua descrição podem ser um ponto de partida para outras investigações. Além disso, percebe-se que a dissonância cognitiva possui um caráter interdisciplinar, podendo ser aplicada em diferentes áreas do conhecimento.

No que diz respeito às implicações gerenciais, a compreensão dessa lacuna existente entre atitude e comportamento e a maneira de como as pessoas lidam com ela, pode ser uma vantagem competitiva, considerando que a preocupação com o consumo sustentável está cada vez mais presente no contexto empresarial. Além disso, pode servir de suporte às empresas para formular ações de marketing e ofertar produtos sustentáveis com maior valor agregado aos seus clientes. Entender o comportamento do consumidor permite criar objetivos organizacionais alinhados com a proteção do meio ambiente e com a responsabilidade social e, ao mesmo tempo, também possibilita influenciar o comportamento de consumo (Gordon, 2013; Peattie \& Peattie, 2009). 
Diante dos resultados deste artigo, um possível caminho para futuras pesquisas seria o de investigar se as variáveis identificadas se aplicam também em outros contextos relacionados ao consumo sustentável. Além disso, pesquisas futuras podem analisar o papel da influência social e sua ligação direta com as normas morais como mediadoras do comportamento de consumo sustentável. Podese examinar também como a relação entre as informações sobre produtos sustentáveis e feedback acontecem, visto que são variáveis semelhantes. Outra possibilidade é avaliar como o comprometimento assumido pelo consumidor relaciona-se à sua atitude e a busca pela recompensa quando a mudança de comportamento ocorre ou não ocorre. Ademais, pode-se investigar como a negação e supressão de culpa, compensação e justificação permitem manter as atitudes intactas, mas comportamentos divergentes. Por fim, pode-se destacar como limitação da presente pesquisa o fato de ter tomado apenas uma base de dados para selecionar os artigos para esta análise.

\section{Referências}

Beretti, A., Figuières, C., \& Grolleau, G. (2013). Behavioral innovations: The missing capital in sustainable development?. Ecological Economics, 89, 187-195.

Bodur, H. O., Duval, K. M., \& Grohmann, B. (2015). Will You Purchase Environmentally Friendly Products? Using Prediction Requests to Increase Choice of Sustainable Products. Journal of Business Ethics, 129(1), 59-75.

Brook, A. (2011). Ecological footprint feedback: Motivating or discouraging?. Social Influence, 6(2), 113-128.

Cohen, S. A., Higham, J. E., \& Cavaliere, C. T. (2011). Binge flying: Behavioural addiction and climate change. Annals of Tourism Research, 38(3), 1070-1089.

Division for Sustainable Development. Disponível em:https://sustainabledevelopment.un.org/topics/sustainableconsumptionandprod uction

Festinger, L. (1957). A theory of cognitive dissonance: Stanford Univ. Pr.

Gordon, R. (2013). Unlocking the potential of upstream social marketing. European Journal of Marketing, 47(9), 1525-1547.

Harmon-Jones, E. (2012) Cognitive Dissonance Theory. The Encyclopedia of Human Behavior, 1, 543-549. 
Harmon-Jones, E., \& Harmon-Jones, C. (2007). Cognitive dissonance theory after 50 years of development. Zeitschrift für Sozialpsychologie, 38(1), 7-16.

Jalonen, H. (2012). The uncertainty of innovation: a systematic review of the literature. Journal of Management Research, 4(1).

Juvan, E., \& Dolnicar, S. (2014). The attitude-behaviour gap in sustainable tourism. Annals of Tourism Research, 48, 76-95.

Kimura, K., \& Shinoki, M. (2007). Decision and Justification in the Social Dilemma of Recycling. I. Sociological Theory and Methods, 22(1), 31-48.

Kovács, J., Pantya, J., Medves, D., Hidegkuti, I., Heim, O., \& Bursavich, J. B. (2014). Justifying environmentally significant behavior choices: An American-Hungarian cross-cultural comparison. Journal of Environmental Psychology, 37, 31-39.

Lavergne, K. J., \& Pelletier, L. G. (2015). Predicting individual differences in the choice of strategy to compensate for attitude-behaviour inconsistencies in the environmental domain. Journal of Environmental Psychology, 44, 135-148.

Liang, Y. (2016). Reading to make a decision or to reduce cognitive dissonance? The effect of selecting and reading online reviews from a post-decision context. Computers in Human Behavior. http://doi.org/10.1016/..chb.2016.07.016

MacDonald, E. F., \& She, J. (2015). Seven cognitive concepts for successful ecodesign. Journal of Cleaner Production, 92, 23-36.

Martinsson, J., \& Lundqvist, L. J. (2010). Ecological citizenship: coming out 'clean'without turning 'green'?. Environmental politics, 19(4), 518-537.

Obradovich, N., \& Guenther, S. M. (2016). Collective responsibility amplifies mitigation behaviors. Springer, 137(1-2), 307-319.

Osbaldiston, R., \& Schott, J. P. (2012). Environmental sustainability and behavioral science: Meta-analysis of proenvironmental behavior experiments. Environment and Behavior, 44(2), 257-299.

Peattie, K., \& Peattie, S. (2009). Social marketing: A pathway to consumption reduction?. Journal of Business Research, 62(2), 260-268.

Petticrew, M., \& Roberts, H. (2008). Systematic reviews in the social sciences: A practical guide. John Wiley \& Sons.

Rashid, N. R. N. A., \& Mohammad, N. (2012). A discussion of underlying theories explaining the spillover of environmentally friendly behavior phenomenon. Procedia-Social and Behavioral Sciences, 50, 1061-1072. 
Rodrigues, A. (1969). Consistência cognitiva e comportamento social. Arquivos Brasileiros de Psicologia Aplicada, 21(2), 9-86.

Rubens, L., Gosling, P., Bonaiuto, M., Brisbois, X., \& Moch, A. (2015). Being a hypocrite or committed while I am shopping? A comparison of the impact of two interventions on environmentally friendly behavior. Environment and Behavior, $47(1), 3-16$.

Schultz, P. W. (2014). Strategies for promoting proenvironmental behavior lots of tools but few instructions. European Psychologist, 19, 107-117

Sottile, E., Meloni, I., \& Cherchi, E. (2015). A hybrid discrete choice model to assess the effect of awareness and attitude towards environmentally friendly travel modes. Transportation Research Procedia, 5, 44-55.

Stenman, J. O., \& Martinsson, P. (2006). Honestly, why are you driving a BMW?. Journal of Economic Behavior \& Organization, 60(2), 129-146.

Tanford, S., \& Montgomery, R. (2015). The effects of social influence and cognitive dissonance on travel purchase decisions. Journal of Travel Research, 54(5), 596610.

Tobler, C., Visschers, V. H., \& Siegrist, M. (2012). Addressing climate change: Determinants of consumers' willingness to act and to support policy measures. Journal of Environmental Psychology, 32(3), 197-207.

Thogersen, J. (2004). A cognitive dissonance interpretation of consistencies and inconsistencies in environmentally responsible behavior. Journal of Environmental Psychology, 24(1), 93-103.

Truelove, H. B., Carrico, A. R., Weber, E. U., Raimi, K. T., \& Vandenbergh, M. P. (2014). Positive and negative spillover of pro-environmental behavior: An integrative review and theoretical framework. Global Environmental Change and Policy Dimensions, 29, 127-138.

Truelove, H. B., Yeung, K. L., Carrico, A. R., Gillis, A. J., \& Raimi, K. T. (2016). From plastic bottle recycling to policy support: An experimental test of pro-environmental spillover. Journal of Environmental Psychology, 46, 55-66.

Verplanken, B., \& Roy, D. (2016). Empowering interventions to promote sustainable lifestyles: Testing the habit discontinuity hypothesis in a field experiment. Journal of Environmental Psychology, 45, 127-134.

Whitmarsh, L., \& O'Neill, S. (2010). Green identity, green living? The role of proenvironmental self-identity in determining consistency across diverse proenvironmental behaviours. Journal of Environmental Psychology, 30(3), 305-314. 